\title{
Efeitos da adaptação às próteses auditivas na qualidade de vida, no equilíbrio e no medo de queda em idosos com perda neurossensorial
}

\section{Effects of hearing aids in the balance, quality of life and fear to fall in elderly people with sensorineural hearing loss}

\author{
Clara Fonseca Lacerda', Luciana Oliveira e Silva², Roberto Sérgio de Tavares Canto ${ }^{3}$, Nadia Carla Cheik4. \\ 1) Mestre em Fisioterapia. Fisioterapeuta da Prefeitura Municipal de Patos de Minas. \\ 2) Mestre em Fisioterapia pelo Centro Universitário do Triângulo (UNITRI). Docente. \\ 3) Pós-Doutorado pela Universidade de Liverpool, Inglaterra (1984) Atuação em Cirurgia, com ênfase em Cirurgia Traumatológica Doutor. Docente do Centro Universitário \\ do Triângulo, Brasil. \\ 4) Doutorado em Ciências Fisiológicas pela Universidade Federal de São Carlos, Brasil (2005). Professora da Universidade Federal de Uberlândia / Faculdade de Educação \\ Física (FAEFI/ UFU). Programa de Mestrado em Fisioterapia pelo Centro Universitário do triângulo (UNITRI). \\ Instituição: Centro Universitário do triângulo (UNITRI). \\ Uberlândia / MG - Brasil. \\ Endereço para correspondência: Programa de Educação Física FAEFI/UFU - Rua Benjamim Constant, 1.286 - Bairro: Aparecida - Uberlândia / MG - Brasil - CEP: \\ 38400-902 - Telefones: (+55 34) 3822-1096 e 9975-4811 - E-mail: clara_fisio@ hotmail.com \\ Artigo recebido em 13 de agosto de 2009. Artigo aprovado em 18 de dezembro de 2011
}

\section{RESUMO}

Introdução: $O$ processo de envelhecimento provoca modificações estruturais e funcionais à saude, comprometendo o controle postural e processamento central. Estudos têm abordado a necessidade de identificar os fatores de risco prejudiciais à saúde auditiva e segurança em idosos acometidos por déficits auditivos e com alterações de equilíbrio.

Objetivo: Avaliar o efeito da prótese auditiva na qualidade de vida, no equilíbrio e no medo de queda em idosos com perda auditiva bilateral.

Método: Estudo clínico e experimental realizado com 56 idosos com perda auditiva neurossensorial, submetidos ao uso da prótese auditiva de amplificação sonora individual (AASI). Os idosos responderam aos questionários de qualidade de vida Short Form Health Survery (SF-36), Falls Efficacy ScaleInternacional (FES-I) e o teste de Berg Balance Scale (BBS). Após 4 meses, os idosos que adaptaram ao uso da AASI foram reavaliados.

Resultados: Houve $50 \%$ de adaptação dos idosos ao AASI. Foi observado que o sexo masculino teve maior dificuldade em adaptar ao aparelho auditivo e que as variáveis idade, grau de perda, presença de zumbido e vertigem não interferiram na adaptação à prótese auditiva. Houve melhora da qualidade de vida nos domínios Estado da Saúde Geral (EGS) e Capacidade Funcional (CF) e do zumbido, assim como o aumento da auto-confiança após adaptação da prótese auditiva.

Conclusão: $O$ uso de prótese auditiva propiciou a melhora dos domínios da qualidade de vida, o que refletiu em uma melhor auto-confiança e consequentemente a longo prazo na redução do medo de queda em idosos com perda auditiva neurossensorial.

Palavras-chave: perda auditiva, qualidade de vida, equilíbrio postural.

\section{SUMMARY}

Introduction: The aging process provokes structural modifications and functional to it greets, compromising the postural control and central processing. Studies have boarded the necessity to identify to the harmful factors of risk to aged the auditory health and security in stricken aged by auditory deficits and with alterations of balance.

Objective: To evaluate the effect of auditory prosthesis in the quality of life, the balance and the fear of fall in aged with bilateral auditory loss.

Method: Carried through clinical and experimental study with 56 aged ones with sensorineural auditory loss, submitted to the use of auditory prosthesis of individual sonorous amplification (AASI). The aged ones had answered to the questionnaires of quality of life Short Form Health Survey (SF36), Falls Efficacy International Scale- (FES-I) and the test of Berg Balance Scale (BBS). After 4 months, the aged ones that they adapted to the use of the AASI had been reevaluated. Results: It had $50 \%$ of adaptation of the aged ones to the AASI. It was observed that the masculine sex had greater difficulty in adapting to the auditory device and that the variable age, degree of loss, presence of humming and vertigo had not intervened with the adaptation to auditory prosthesis. It had improvement of the quality of life in the dominance of the State General Health (EGS) and Functional Capacity (CF) and of the humming, as well as the increase of the auto-confidence after adaptation of auditory prosthesis.

Conclusion: The use of auditory prosthesis provided the improvement of the domains of the quality of life, what it reflected consequently in one better auto-confidence and in the long run in the reduction of the fear of fall in aged with sensorineural auditory loss.

Keywords: hearing loss, quality of life, postural balance. 


\section{INTRODUÇÃO}

No idoso, a perda auditiva, é uma das três condições crônicas mais prevalentes, ficando atrás somente da artrite e da hipertensão $(1,2)$. Segundo a (3), aproximadamente $90 \%$ das pessoas com idade superior a 80 anos apresentam perda auditiva.

Quando ocorre em função do processo de envelhecimento é conhecida como presbiacusia e gera um dos mais incapacitantes distúrbios da comunicação, impedindo os idosos desempenharem a sociedade, porque não só provoca a privação sensorial de ouvir, como a dificuldade de compreensão da fala daqueles que o cercam dificultando a plena comunicação $(1,4,5,6)$. Além disso, acarreta uma série de problemas sociais, dentre eles: o afastamento das atividades sociais e familiares, baixa auto-estima, isolamento, solidão, depressão e irritabilidade $(1,7,8)$. O processo de envelhecimento não representa somente a perda do limiar auditivo, ele gera modificações estruturais e funcionais comprometendo todos os componentes do controle postural, sensorial (visual, somatossensorial e vestibular), efetor (força, amplitude de movimento, alinhamento biomecânico, flexibilidade) e o processamento central $(9,10)$.

A diminuição fisiológica da visão, da audição, da estabilidade corporal, as alterações articulares e da potência muscular, podem facilitar os riscos de acidentes e queda pela lentificação das reações defensivas (11).

As quedas são tratadas como fator de grande relevância epidemiológica, social e econômica em todo o mundo, pois, é o tipo mais comum de acidente entre os idosos (12). As complicações lideram as causas de mortes em pessoas acima de 65 anos e promove deficiência física, psicológica, e social, podendo levar o indivíduo a dependência, redução das atividades diárias e da confiança, alteração do estilo de vida (13) gerando consequências negativas em relação à qualidade de vida (14).

Como a expectativa de vida está aumentando, é importante adotar medidas nas quais minimizem os prejuízos causados por ela uma vez que são raras as indicações cirúrgicas e medicamentosas, prevalecendo o uso de adaptações de aparelho de amplificação sonora individual (AASI). O uso permite o resgate da percepção dos sons da fala, além dos sons a mbientais, promovendo a melhora da habilidade de comunicação (15).

O aumento da visibilidade social do segmento de pessoas com mais de 60 anos, no Brasil, foi um dos fatores que instigaram a mobilização de órgãos governamentais e não governamentais para o atendimento das novas deman- das surgidas no âmbito da saúde, da assistência e da seguridade social (16). Em 2004 o Ministério da Saúde, instituiu a Política Nacional de Atenção à Saúde Auditiva e através da portaria SAS/MS nº. 587 houve distribuição da rede estadual para ações na atenção básica, na média e alta complexidade (BRASIL, Ministério da Saúde, 2004). Por meio da deliberação CIB-SUS-MG no 156 em 21/03/2005, a Associação de Pais e Amigos dos Excepcionais (APAE) de Patos de Minas foi credenciada e habilitada à prestação de serviço do referido programa garantindo ao indivíduo o diagnóstico, adaptação e fornecimento do aparelho de amplificação sonora individual - AASI (17).

Neste contexto, o objetivo deste estudo foi avaliar equilíbrio, medo de queda e qualidade de vida de idosos com perda auditiva neurossensorial bilateral antes e após a adaptação ao aparelho de amplificação sonora individual (AASI).

\section{MÉTODO}

Este estudo foi previamente aprovado pelo Comitê de Ética e Pesquisa com seres humanos da UNITRI (registro $\mathrm{n}^{\mathrm{o}}$ 647923) e seguiu a resolução 196/96 do Conselho Nacional de Saúde. Todos os procedimentos foram explicados como também descritos detalhadamente no termo de consentimento, que foi assinado pelos voluntários, e a partir de então, iniciou-se a coleta de dados.

A amostra foi constituída por 56 indivíduos (32 do sexo masculino) entre 60 e 84 anos, portadores de perda auditiva neurossensorial bilateral, selecionados a partirdos prontuários do Serviço de Atençãoà Saúde Auditiva (SASA).

Os prontuários foram constituídos por avaliação do otorrinolaringologista, avaliaçãoaudiológica, fonoaudiológica, entrevista com assistente social e psicólogo. Todos os voluntários foram protetizados com aparelhos retroauriculares.

Foram incluídos os idosos portadores de perda auditiva neurossensorial bilateral inseridos no Sistema de Atenção à Saúde Auditiva (SASA) de Patos de Minas/ MG com idade entre 60 e 84 anos; e excluídos os usuários de prótese ou órtese de membros inferiores; portadores de distúrbios neurológicos e comportamentais; indivíduos previamente submetidos à reabilitação auditiva; não assinatura do Termo de Consentimento Livre e Esclarecido; não compreensão dos comandos para a aplicação dos testes.

Na avaliação foram coletados os dados de identificação, clínicos, antropométricos (peso, altura e índice de massa corpórea - IMC). 
Os voluntários responderam aos questionários de qualidade de vida (questionário SF-36) (18) e de medo de cair (Falls Efficacy Scale - Internacional /FES-I) (19) e realizaram o teste de equilíbrio (Berg Balance Scale/BBS) (20). Após 4 meses, os voluntários que adaptaramao AASI, retornaram para reavaliação, refizeram o teste de equilíbrio, e responderam além dos questionários citados ao Satisfaction with Amplification in Daily Life(SADL) (21). Todos os questionários aplicados nesta pesquisa foram previamente validados e possuem propriedades psicométricas adequadas.

\section{Análise estatística}

A prevalência das variáveis: sexo, grau de perda auditiva, presença de zumbido, presença de vertigem, da amostra foram dicotomizadas e analisadas pelo teste do Qui-quadrado de independência.

Para análise comparativa dos escores dos domínios do SF-36 foi utilizado o teste de Mann Whitney. O teste T foi utilizado para comparar os dados antropométricos e idade da amostra.

Os valores de FES-I, BERG e BBS entre os grupos adaptados, não-adaptados e reavaliados foram comparados por meio da Análise da Variância (ANOVA Two Way) e o teste de Post-hoc de Tukey.

Para correlacionar os escores dos domínios do SF-36, a escala BBS, ao FES-I, à presença de vertigem, à presença de zumbido e à idade dos voluntários foi usada a Correlação de Spearman.

Foi considerado um nível de significância de 5\% $(\mathrm{p}<0,05)$ em todos os testes.

\section{RESULTADOS}

Nota-se na Tabela 1, uma distribuição homogênea entre os sexos e quanto ao grau de perda auditiva e uma alta prevalência de zumbido e vertigem entre os participantes.

A Tabela 2 apresenta as características antropométricas, idade e escore das escalas FES-I e BBS dos voluntários. Verifica-se sobrepeso na amostra, bom nível de equilíbrio e pouco medo de cair.

Observamos que os voluntários apresentam bons escores nos domínios do SF-36, o que indica que não há redução na qualidade de vida em idosos com perda auditiva, entretanto destaca-se redução do item vitalidade (Tabela 3).
Após 4 meses da colocação do a parelho auditivo os voluntários que se adaptaram a o aparelho auditivo foram reavaliados. Em nosso estudo, 50\% dos voluntários adaptaram ao aparelho auditivo. Na Tabela 4, nota-se uma distribuição homogênea das variáveis antropométricas e idade entre os grupos.

Tabela I. Caracterização Geral da Amostra N=56.

\begin{tabular}{ll}
\hline Variáveis & \multicolumn{1}{c}{$N(\%)$} \\
\hline Sexo masculino & $32(57 \%)$ \\
Graude perda & $15(27 \%)$ \\
Perda Moderada & $21(37 \%)$ \\
Perda Severa & $20(36 \%)$ \\
Perda Profunda & $39(69,6 \%)$ \\
Presença de zumbido & $32(57 \%)$ \\
Presença de vertigem &
\end{tabular}

Valor absoluto (valor percentual)

Tabela 2. Características antropométricas, idade e escore das escalas FES-I e BBS dos voluntários $(\mathrm{N}=56)$.

\begin{tabular}{ll}
\hline Variáveis & Média \pm DP \\
\hline Massa Corporal & $69,0 \pm 6,7$ \\
IMC $\left(\mathrm{Kg} / \mathrm{m}^{2}\right)$ & $27,4 \pm 4,9$ \\
Idade & $72,1 \pm 6,7$ \\
FES-I & $25,8 \pm 7,8$ \\
BBS & $51,3 \pm 5,1$ \\
\hline
\end{tabular}

Os dados estão apresentados em média e desvio padrão.

Tabela 3. Média e desvio padrão dos domínios do SF-36 $(\mathrm{N}=56)$.

\begin{tabular}{lc}
\hline Domínios SF-36 & Média \pm DP \\
\hline CF & $64 \pm 23,9$ \\
AF & $83,5 \pm 29,2$ \\
DOR & $67,1 \pm 24,3$ \\
EGS & $72,5 \pm 17,2$ \\
VIT & $50,7 \pm 14,0$ \\
AS & $83,5 \pm 22,8$ \\
AE & $72,3 \pm 42,6$ \\
SM & $54,4 \pm 10,9$
\end{tabular}

Os dados estão apresentados em média e desvio padrão. CF, Capacidade Funcional; AF, Aspecto Físico; EGS, Estado Geral de Saúde; VIT, Vitalidade; AS, Aspectos Sociais; AE, Aspectos Emocionais; SM, Saúde Mental.

Tabela 4. Características antropométricas da amostra separadapor grupos deidosos:Adaptados(A)eNãoAdaptados (NA).

\begin{tabular}{lcc}
\hline Variáveis & $\mathrm{A}(\mathrm{n}=28)$ & $\mathrm{NA}(\mathrm{n}=28)$ \\
\hline Massa Corporal $(\mathrm{Kg})$ & $68,1 \pm 15,4$ & $69,6 \pm 12,1$ \\
Estatura $(\mathrm{m})$ & $1,6 \pm 0,1$ & $1,6 \pm 0,1$ \\
$\mathrm{IMC}\left(\mathrm{kg} / \mathrm{m}^{2}\right)$ & $27,6 \pm 5$ & $27,2 \pm 4,8$ \\
Idade & $70,6 \pm 6,6$ & $73,6 \pm 6,7$ \\
\hline
\end{tabular}

Os dados estão apresentados em média e desvio padrão. 
Quanto à adaptação ao AASI, nota-se que o sexo masculino teve maior dificuldade em adaptar ao aparelho auditivo e que o grau de perda auditiva, queixas de zumbido e vertigem, são variáveis que não influenciaram na adaptação do AASI (Tabela 5).

Como apontado na Tabela 6 , em relação a queixa de zumbido, apenas 4 dos 20 voluntários não referiram benefícios com o uso da prótese auditiva nesta variável. Quanto ao questionário SADL, no item referente a autoconfiança, $100 \%$ dos voluntários relataram melhora desta variável após adaptação, sendo que 90\% responderam muita melhora da autoconfiança.

Na Tabela 7 observa-se que os idosos que não se adaptaram apresentam maior capacidade funcional que os idosos do grupo adaptado. Entretanto, na reavaliação do grupo adaptado (RA), verificou-se uma melhora significativa ao comparar com a avaliação. Além disto, houve

Tabela 5. Características gerais da amostra dos idosos avaliados: Adaptados (A) e Não Adaptados (NA).

\begin{tabular}{|c|c|c|}
\hline Variáveis & $A(n=28)$ & $N A(n=28)$ \\
\hline Sexo & $13(47 \%)$ & $19(68 \%)$ \\
\hline $\begin{array}{l}\text { Masculino } \\
\text { Feminino }\end{array}$ & 15 (53\%) & $9(32 \%)$ \\
\hline GraudePerdaAuditiva & - & - \\
\hline Leve & $8(28,6 \%)$ & 7 (25\%) \\
\hline Moderada & $10(35,7 \%)$ & || (39\%) \\
\hline $\begin{array}{l}\text { Severa } \\
\text { Profunda }\end{array}$ & $10(35,7 \%)$ & $10(36 \%)$ \\
\hline Vertigem & $16(57 \%)$ & $16(57 \%)$ \\
\hline $\begin{array}{l}\text { Comvertigem } \\
\text { Semvertigem }\end{array}$ & $12(43 \%)$ & $12(43 \%)$ \\
\hline $\begin{array}{l}\text { Zumbido } \\
\text { Comzumbido } \\
\text { Semzumbido }\end{array}$ & $\begin{array}{l}20(71,4 \%) \\
8(28,6 \%)\end{array}$ & $\begin{array}{l}19(67,8 \%) \\
9(32,2 \%)\end{array}$ \\
\hline
\end{tabular}

Valorabsoluto(valor percentual).

Tabela 6. Comparação após 4 meses de adaptação ao AASI quanto a melhora do zumbido e da auto confiança.

\begin{tabular}{lcc}
\hline Variável & Adaptados(A) & Reavaliados(RA) \\
& $N=28$ & $N=28$ \\
& Antes do AASI & Após 4 meses \\
& do AASI \\
\hline ComZumbido & $20(79 \%)$ & $4(20 \%)$ \\
Auto confiança & - & $28(100 \%)$ \\
\hline
\end{tabular}

Valor absoluto (valor percentual). melhora significativa do EGS do grupo adaptado ao compararmos os valores da avaliação e reavaliação.

Na análise intergrupo, não houve diferença entre os escores da BBS e da FES-I, no entanto, na análise intragrupo, o grupoadaptadoapresentou menor medode cair(Tabela 8).

A Tabela 9 apresenta as correlações obtidas dos grupos avaliados entre os domínios do SF-36 e os escores das escalas FES-I, BBS, presença de vertigem, presença de zumbido e a idade. Verifica-se que no grupo adaptado houve correlação positiva entre a BBS e os domínios CF, DOR, EGS e AS e uma correlação negativa entre a FES-I e os domínios CF e DOR.

Após a adaptação do AASI, nota-se correlação positiva entre a BBS e os domínios CF e EGS, bem como, entre idade AE e AS. Nota-se uma correlação negativa entre medo de queda e os domínios CF, EGS e VIT.

Tabela 7. Resultados da avaliação da qualidade de vida dos grupos avaliados. Os valores estão expressos em média e desvio padrão.

\begin{tabular}{lccr}
\hline $\begin{array}{l}\text { Domínios } \\
\text { SF-36 }\end{array}$ & $\mathrm{A}(\mathrm{n}=28)$ & $\mathrm{NA}(\mathrm{n}=28)$ & $\mathrm{RA}(\mathrm{n}=28)$ \\
\hline CF & $57,5 \pm 15$ & $69,3 \pm 19,7 *$ & $71,9 \pm 15+$ \\
AF & $82,8 \pm 30,9$ & $84,3 \pm 28,6$ & $83,5 \pm 29,5$ \\
DOR & $68,9 \pm 24,8$ & $66,9 \pm 23,9$ & $67,1 \pm 24,3$ \\
EGS & $68,7 \pm 18,1$ & $75,6 \pm 15,5$ & $72,5 \pm 17,2+$ \\
VIT & $51,3 \pm 11,6$ & $48,5 \pm 16,7$ & $48,7 \pm 14,0$ \\
AS & $85,3 \pm 23,8$ & $81,5 \pm 22,3$ & $83,5 \pm 22,8$ \\
AE & $72,6 \pm 41,6$ & $75,3 \pm 41,7$ & $72,3 \pm 42,6$ \\
SM & $56,3 \pm 12,6$ & $52,4 \pm 9,2$ & $54,4 \pm 10,9$ \\
\hline
\end{tabular}

CF, Capacidade Funcional; AF, Aspecto Físico; EGS, Estado Geral de Saúde; VIT, Vitalidade; AS, Aspectos Sociais; AE, Aspectos Emocionais; SM, Saúde Mental.

A, Grupo Adaptado; NA, Grupo Não Adaptado; RA, Reavaliação dos Adaptados.

*p<0,05 comparando A X NA

$+\mathrm{p}<0,05$ comparando A X RA

Tabela 8. Escores das escalas FES-le BBS dos grupos avaliados. Os valores estão expressos em média e desvio padrão.

\begin{tabular}{lccc}
\hline Variáveis & $\mathrm{A}(\mathrm{n}=28)$ & $\mathrm{NA}(\mathrm{n}=28)$ & $\mathrm{RA}(\mathrm{n}=28)$ \\
\hline FES-I & $27,7 \pm 8,3$ & $24,5 \pm 7, \mathrm{I}$ & $25,3 \pm 8,0+$ \\
BBS & $50,6 \pm 4,9$ & $51,4 \pm 5,4$ & $51 \pm 5,0$ \\
\hline
\end{tabular}

A, Grupo Adaptado; NA, Grupo Não Adaptado; RA Reavaliação dos Adaptados.

*p $\leq 0,05$ comparando A X NA

$+\mathrm{p}<0,05$ comparando A X R 
Tabela 9. Correlações obtidas dos grupos avaliados entre os domínios do SF-36 e os escores das escalas FES-I, BBS, presença de vertigem, presença de zumbido e a idade.

\begin{tabular}{lcccccc}
\hline Domínios SF-36 & & FES-I & BBS & PresençadeVertigem & Presençade Zumbido & Idade \\
& Grupos & $r$ & $r$ & $r$ & $r$ & $r$ \\
\hline CF & A & $-0,69 *$ & $0,78^{*}$ & 0,23 & 0,22 & $-0,18$ \\
& RA & $-0,64 *$ & $0,54 *$ & 0,32 & 0,2 & $-0,22$ \\
AF & A & $-0,16$ & 0,01 & 0,330 & 0,27 & 0,21 \\
& RA & 0,28 & $-0,31$ & $-0,05$ & $-0,03$ & 0,25 \\
DOR & A & $-0,41 *$ & $0,36 *$ & 0,01 & 0,15 & $-0,06$ \\
& RA & $-0,16$ & 0,05 & 0,28 & $-0,15$ & 0,19 \\
EGS & A & $-0,29$ & $0,35 *$ & 0,15 & 0,08 & $-0,3$ \\
& RA & $-0,50 *$ & $0,52 *$ & 0,17 & $-0,03$ & 0,09 \\
VIT & A & 0,15 & 0,25 & 0,15 & 0,15 & 0,17 \\
& RA & $0,33 *$ & 0,11 & 0,21 & 0,22 & 0,29 \\
AS & A & $-0,25$ & $0,35 *$ & 0,23 & 0,09 & 0,28 \\
& RA & 0,28 & $-0,22$ & $-0,19$ & $-0,12$ & 0,34 \\
AE & A & $-0,16$ & $-0,15$ & 0,04 & 0,11 & $-0,13$ \\
& RA & 0,06 & $-0,17$ & $-0,06$ & $-0,15$ & 0,34 \\
SM & A & $-0,16$ & $-0,15$ & 0,04 & 0,11 & $-0,13$ \\
& RA & $-0,22$ & $-0,06$ & 0,07 & 0,18 & 0,22 \\
\hline
\end{tabular}

CF, Capacidade Funcional;AF, Aspectos Físicos; EGS, Estado Geralde Saúde;VIT, Vitalidade;AS, Aspectos Sociais;AE, Aspectos Emocionais; SM, Saúde Mental; A, Grupo adaptado; NA, Grupo não adaptado, RA, Reavaliação dos adaptados. "p $\leq 0,005$; $+p<0,06 ;{ }^{\circ} p<0,0$

\section{DISCUSSÃO}

No presente estudo teve por finalidade avaliar a contribuição do aparelho auditivo AASI na qualidade de vida, equilíbrio e medo de cair em idosos com perda auditiva neurossensorial bilateral.

Observarmos maior prevalência de perda auditiva em homens (57\%) corroborando com outros estudos (7-8). A queixa de zumbido $(69,6 \%)$ e vertigem $(57 \%)$ associada à perda auditiva foi altamente prevalente na amostra uma vez que processos degenerativos inerentes à idade são responsáveis pela ocorrência de vertigem e zumbido na população geriátrica (10).

Oescore médio do questionário que avalia equilíbrio foi 51 pontos, o que representa um bom equilíbrio funcional, provavelmente pelo fato de que nossa amostra foi constituída por idosos não institucionalizados com perda auditiva e boa capacidade funcional, uma vez que foram excluídos indivíduos que faziam uso de dispositivo de auxílio à marcha e que apresentassem doenças neurológicas.

Quanto ao medo de queda foi observado um escore médio de 25,5 nos voluntários desta pesquisa. Resultados similares foram encontrados por (22) que ao avaliar a FESI de idosos da comunidade e idosos fisicamente ativos encontrou um escore médio de 24,9 e 27,5 respectivamente. Desta forma podemos inferir que, a perda auditiva não influenciou no medo de queda. Provavelmente, isto está relacionado ao bom equilíbrio que os idosos apresentaram o que diminui o medo de queda.

Em geral, as médias dos escores dos domínios do SF36 foram acima de $50 \%$ demonstrando que os voluntários têm boa qualidade de vida. Segundo (23) no SF-36 um escore elevado no domínio aspecto físico e capacidade funcional pode indicar que os idosos possuem pouca limitação ao trabalho e em atividades de vida diária, apresentam boa saúde e habilidade funcional.

Sabe-se que o conceito de qualidade de vida (QV) é muito amplo, subjetivo, dependente do nível sócio-cultural, das ambições pessoais dos indivíduos e não somente da estrutura acometida (24). Isto pode ser observado no presente estudo, pois, nem sempre uma estrutura acometida, como a perda auditiva interfere diretamente na qualidade de vida dos voluntários, sendo a $Q V$ um produto de vários fatores.

Todos os voluntários da amostra foram protetizados. Após 4 meses de uso do AASI, 50\% dos voluntários adaptaram ao aparelho auditivo. Nos voluntários adaptados houve reavaliação das variáveis: qualidade de vida, equilíbrio e medo de queda. Este prazo foi estabelecido em virtude de estudos demonstrarem que 90 dias são suficientes para uma boa 
adaptação e aclimatização do AASI (25). Quanto aos fatores relacionados à adaptação ao AASI, nossos achados estão de acordo com estudo previamente realizado que demonstrou que presença de zumbido e vertigem não influenciam na adaptação ao AASI (26). No entanto, vale salientar que, estudos prévios demonstram que fatores como relato de satisfação com oaparelho, o número de horas/dia, participação da família (5), expectativas, necessidades de comunicação, grau de perda, tolerância para sons intensos, expectativas, motivação (4) e reabilitação auditiva (2), são critérios importantes para uma adaptação bem sucedida.

Uma das principais queixas dos idosos com presbiacusia é a presença do zumbido que frequentemente é um fator de grande repercussão negativa na vida do indivíduo, comprometendo a qualidade de vida, dificultando o sono, a concentração nas atividades diárias e profissionais, assim como a vida social (27). O zumbido é uma desordem extremamente frequente em pacientes com perda auditiva, atingindo cerca de 40 milhões de pessoas nos EUA, afetando aproximadamente $1 / 3$ da população acima dos 65 anos de idade (28). A prática clínica tem demonstrado que indivíduos portadores de perda auditiva associada a zumbido beneficiam-se com o uso de próteses auditivas, pois estas, além de melhorarem a compreensão da conversação, aliviam o zumbido, (29), demonstraram que o uso da prótese auditiva melhorou o zumbido em $78,2 \%$ dos indivíduos e em nossa amostra $79 \%$ dos voluntários adaptados se beneficiaram com redução do zumbido.

De acordo com (6) o uso de prótese auditiva beneficia as atividades cotidianas e a funcionalidade em indivíduos portadores de perda auditiva neurossensorial. Isto corrobora com nossos achados, pois encontramos melhora significativa da capacidade funcional (CF) e do estado geral de saúde (EGS). Provavelmente devido à adaptação ao AASI, ocorreu melhora da audição, da habilidade de comunicação e sentimento de segurança, que também influenciaram o aumento do EGS e CF, e consequentemente melhora da qualidade de vida (7).

Tendo como respaldo os achados de (30) acreditamos que o aumento do EGS resultou em aumento nas atividades de vida diárias e consequentemente da CF o que refletiu em redução do medo de queda dos voluntários. Outro fator associado a esta redução possivelmente foi o aumento da confiança e segurança relatados pelos voluntários a partir dos resultados positivos do questionário SADL.

\section{CONCLUSÃO}

Idosos com perda auditiva neurossensorial apresentaram boa qualidade de vida e equilíbrio e referiram pouca preocupação em cair. Entretanto após adaptação ao AASI, houve melhora da qualidade de vida (EGS e CF), redução do medo de queda e do zumbido e aumento da autoconfiança após adaptação ao AASI. Vale salientar ainda que somente 50\% dos voluntários se adaptaram e que o sexo masculino teve maior dificuldade em adaptar ao aparelho auditivo e que as variáveis idade, grau de perda, presença de zumbido e vertigem, não foram fatores que interferiram na adaptação ao AASI.

A partir deste estudo podemos inferir que tão importante o AASI é para o idoso, quanto é o benefício direto dele na intervenção fisioterápica. O paciente bem adaptado terá maior capacidade de comunicação, aumento da autoconfiança, maior atenção e compreensão das informações que são fatores importantes em uma interação preventiva e reabilitativa. Vale salientar que o desenvolvimento de novos estudos que visem elucidar os fatores associados à adesão ao AASI seria de grande interesse, pois evitaria gastos desnecessários da saúde pública.

\section{REFERÊNCIAS BIBLIOGRÁFICAS}

1. Bess FH, Hedley-Williams A, Lichtenstein MJ. Avaliação Audiológica em idosos. In: Musiek FE; Rintelmann MJ. Perspectivas atuais em Avaliação Auditiva. São Paulo: Manole; 2001. p.343-369.

2. Miranda EC, Andrade AN, Gil D, Iório MCM. A efetividade do treinamentoauditivo formal emidosos usuários e próteses auditivas no período e aclimatização. Rev Soc Bras Fonoaudiol. 2007, 12(4):316-21.

3. Wisconsin Self Help for Hard of Hearing People. 2002. Hearing Loss Statistics. [Acessado 18 de dez 2007] www.wishhh.org/hearing_loss_statistics.htm_.

4. Batista ACM, Sampaio FM. Nível de Satisfação dos Idosos usuários de próteses auditivas doadas pela APAC_NAMI_UNIFOR. Rev Bras em Promoção da Saúde. 2005,18:7-10.

5. Miranda EC, Calais LL, Vieira EP, Carvalho LMMA, Borges CLC, Iório MCM. Dificuldades e benefícios com o uso de prótese auditiva: percepção do idoso e sua família. Rev Soc Bras Fonoaudiol. 2008, 13(2):166-72.

6. Silman S, Iório MCM, MizhahI MM, Parra VM. Próteses auditivas: um estudo sobre seu benefício na qualidade de vida de indivíduos portadores de perda auditiva neurossensorial. Distúrbios da Comunicação. 2004, 16(2):153-165.

7. Fausti SA, WIlmington D, Helt PV, Helt, WJ, Konrad-Martin D. Hearing health and care: The need for improved hearing 
loss prevention and hearing conservation practices-Journal of Rehabilitation Research \& Development. 2005, 42(4):45-62.

8. Teixeira AR, Thedy RB, Jotz G. Barba MC. Sintomatologia depressiva em deficientes auditivos adultos e idosos: importância do uso de próteses auditivas. Arq Int Otorinolaringol. 2007, 11:453-458.

9. Chandler JM. Equilíbrio e quedas no idoso: questões sobre a avaliação e o tratamento. In: Guccione AA. Fisioterapia Geriátrica. 2.ed. Rio de Janeiro: Guanabara Koogan; 2002. p.265-77.

10. Ruwer SL, Rossi AG, Simon LF. Equilíbrio no idoso. Rev Bras Otorrinolaringol. 2005, 71(3):298-303.

11. Pereira AMM. A queda e suas conseqüências para o idoso: Aspectos psicológicos e emocionais. [Dissertação]. Uberlândia: Universidade Federal de Uberlândia; 2006.

12. RebelattoJR, Castro AP, Sako FK, Aurichio TR. Equilíbrio estático e dinâmico em indivíduos senescentes e o índice de massa corporal. Fisioterar Mov. 2008, 21(3):69-75.

13. Maciel ACC, Guerra RO. Fatores associados à alteração da mobilidade em idosos residentes na comunidade. Revista Brasileira de Fisioterapia 2005, 9(1):17-23.

14. Ribeiro AP, Souza ER, Atie S, Souza AC, Schilithz AO. Influência das quedas na qualidade de vida de idosos Ciência \& Saúde Coletiva. 2008, 13(4):1265-1273.

15. Magni C, Freilberger F, Tonn K. Avaliação do grau de satisfação entre os usuários de amplificação de tecnologia analógica e digital. Rev Bras Otorrinolaring. 2005; 71(5): 650-657.

16. Rodrigues NC, Rauth J. Os Desafios do Envelhecimento no Brasil In: Freitas et al. Tratado de Geriatria e Gerontologia, Rio de Janeiro: Guanabara Koogan; 2002. p.187-192.

17. Brasil. Ministério da Saúde. In Deliberação CIB-SUS-MG N. ${ }^{\circ} 156$, de 21 de março de 2005. Credencia a Unidade de Serviço de Atençãoà Saúde Auditiva, Associação de Pais e Amigos dos Excepcionais - APAE, do município de Patos de Minas. [Acessado em: 04 de abril 2006]. Disponível http:/ /www.saude.mg.gov.br/atos_normativos/deliberacoes/ 2005/del_ 156pdf

18. Ware JE, Sherbourne CD. The MOS 36 item shortform health survey (SF36): I. conceptual framework and item selection. Med Care. 1992, 30:473-83.

19. Yardley L, Beyer N, Hauer K, Kempen G, Piot-Ziegler C, Todd C. Development and initial validation of the falls efficacy scale-international (FES-I). Age and Ageing. 2005, 34:614-9.

20. Berg KO, MAKI BE, Willians JI, Holliday PJ, WoodDauphinee SL. Clinical and laboratory measures of postural balance in an elderly population. Arch Phys Med Rehabil. 1992, 73:1073-1080.

21. Cox RM, Alexander GC. Measuring satisfaction with amplification in daily life: The SADL scale. Ear Hear 1999; 20(4):306-20.

22. Narciso FV. Avaliação do equilíbrio e da altura percentual do centro de gravidade de idosos. [Dissertação]. Uberlândia: Mestrado em Fisioterapia do Centro Universitário do Triângulo; 2008.

23. Santos LS. Influencia da atividade física na qualidade de vida e no escore de ansiedade e depressão em idosos nãoinstitucionalizados. [Dissertação]. Uberlândia: Mestrado em Fisioterapia do Centro Universitário do Triângulo; 2008.

24. Vecchia RD, Ruiz T, Bocchi SCM, Corrente JE. Qualidade de vida na terceira idade: um conceito subjetivo. Rev Bra Epidemiol. 2005, 89(3):246-52.

25. Prates LPCS, Iório MCM. Aclimatização: estudo do reconhecimento de fala em usuários de próteses auditivas. Barueri, Pró-Fono R Atual Cient. 2006, 18(3)259-66.

26. Mulrow CD, Tuley MR, Aguilar C. Correlates successful hearing aid use in olders adults. Ear and Hearing. 1992, 13(2):108-113.

27. Silva RCF, Bandini, HHM, Soares IA. Aparelho de amplificação sonora individual: melhora a sensação de zumbido? Rev CEFAC. 2007, 9(2):263-68.

28. Lynn SG, Bauch CD, Williams DE, Beatty CW, Mellon W, Weaver AL. Psychologic profile of tinnitus patients using the SCL-90-R and tinnitus handicap inventory. Otol Neurotol 2003, 24(6):878-881.

29. Moura LO, Iório MC, AzevedoMF. A eficácia da adaptação de prótese auditiva na redução ou eliminação do zumbido Rev Bras Otorrinolaringol. 2004, 70(5):624-31.

30. Zijlstra GAR, Haastregt JCM, Eijk MY, Rossum V, Stalenhoef PA, kempen JM. Prevalence and correlates of fear of falling, and associated avoidance of activity in the general population of community-living older people. Age and ageing. 2007, 36:304-309. 